\title{
Solvability of boundary value problems for impulsive fractional differential equations in Banach spaces
}

Fang Li ${ }^{*}$ and Huiwen Wang

"Correspondence:

fangli860@gmail.com

School of Mathematics, Yunnan

Normal University, Kunming,

650092, P.R. China

\begin{abstract}
Of concern is the existence of solutions for a class of boundary value problems for impulsive fractional differential equations involving the Caputo fractional derivative in a Banach space. Our approach is based upon the techniques of noncompactness measures and the fixed point theory. Two examples are presented to illustrate the results.
\end{abstract}

MSC: 34A08; 34A37; 34K30; 47H08

Keywords: boundary value problem; fractional differential equations; impulsive condition; measure of noncompactness

\section{Introduction}

The fractional differential equations have received increasing attention during recent years and have been studied extensively (see, e.g., [1-8] and references therein). This is mostly due to the fact that fractional calculus provides an efficient and excellent instrument to describe many practical dynamical phenomena which arise in viscoelasticity, electrochemistry, control, porous media, electromagnetic, etc.

A strong motivation for studying impulsive fractional differential equations comes from the fact they can be used to model phenomena that cannot be modeled by traditional initial value problems, such as the dynamics of populations subject to abrupt changes (harvesting, diseases, etc.) and mechanics, electrical engineering, medicine biology, ecology, and so on. We refer the readers to [9-15] for the general theory and applications of impulsive differential equations. Recently, Ahmad et al. [14] applied the measure of noncompactness to a class of impulsive integrodifferential equations in a Banach space. Liu and Ahmad [15] discussed the existence and uniqueness of solutions for initial value problems of nonlinear singular multiterm impulsive Caputo type fractional differential equations on the half line.

Moreover, some researchers (see [16-20] and the references therein) have addressed the theory of boundary value problems for impulsive fractional differential equations. However, to the best of our knowledge, few papers can be found in the literature for the impulsive fractional differential equations with boundary value conditions in abstract spaces.

2014 Li and Wang: licensee Springer. This is an Open Access article distributed under the terms of the Creative Commons Attribution License (http://creativecommons.org/licenses/by/2.0), which permits unrestricted use, distribution, and reproduction in any medium, provided the original work is properly cited. 
In this paper, we study the following fractional impulsive differential equations with boundary value conditions in a Banach space $X$ :

$$
\begin{aligned}
& { }^{c} D_{t}^{q} u(t)=f(t, u(t)), \quad t \in J^{\prime}:=J \backslash\left\{t_{1}, t_{2}, \ldots, t_{m}\right\}, J=[0,1], \\
& \Delta u\left(t_{k}\right)=I_{k}\left(u\left(t_{k}\right)\right), \quad \Delta u^{\prime}\left(t_{k}\right)=\widetilde{I}_{k}\left(u\left(t_{k}\right)\right), \quad k=1,2, \ldots, m, \\
& u(0)+u(1)=\alpha \int_{0}^{1} q_{1}(u(s)) d s, \\
& u^{\prime}(0)+u^{\prime}(1)=\beta \int_{0}^{1} q_{2}(u(s)) d s,
\end{aligned}
$$

where $1<q<2, \alpha, \beta \in \mathbf{R}$. The fractional derivative is understood here in the Caputo sense. Here $f: J \times X \rightarrow X, I_{k}, \widetilde{I}_{k}: X \rightarrow X(k=1,2, \ldots, m), q_{1}, q_{2}: X \rightarrow X$ are appropriate functions to be specified later. The impulsive moments $\left\{t_{k}\right\}$ are given such that $0=t_{0}<t_{1}<\cdots<t_{m}<$ $t_{m+1}=1, \Delta u\left(t_{k}\right)$ represents the jump of function $u$ at $t_{k}$, which is defined by $\Delta u\left(t_{k}\right)=u\left(t_{k}^{+}\right)-$ $u\left(t_{k}^{-}\right)$, where $u\left(t_{k}^{+}\right), u\left(t_{k}^{-}\right)$represent the right and left limits of $u(t)$ at $t=t_{k}$, respectively.

Observe that problem (1.1)-(1.4) reduces to an anti-periodic boundary value problem in [21] for $\alpha=0$ and $\beta=0$. If $\alpha \neq 0$ or $\beta \neq 0$, then it reduces to an integral boundary problem.

The rest of this paper is organized as follows. In Section 2, we state some basic concepts, notations and preliminary results about fractional calculus and measure of noncompactness. In Section 3, we discuss a new existence result (Theorem 3.1) for solutions of problem (1.1)-(1.4) on $X$, and obtain the corresponding result (Theorem 3.2) in $\mathbf{R}$ immediately. We shall give two illustrative examples for our results in Section 4.

\section{Preliminaries}

Throughout this paper, we denote by $X$ a separable Banach space with norm $\|\cdot\|$. For measurable functions $\xi: J \rightarrow X$, define the norm

$$
\|\xi\|_{L^{p}}=\left(\int_{J}\|\xi(t)\|^{p} d t\right)^{\frac{1}{p}}, \quad 1 \leq p<\infty .
$$

Let $L^{p}(J, X)$ be the Banach space of all Lebesgue measurable functions $\xi: J \rightarrow X$ with $\|\xi\|_{L^{p}}<\infty$.

Let $J_{0}=\left[0, t_{1}\right], J_{k}=\left(t_{k}, t_{k+1}\right], k=1,2, \ldots, m, P C(J, X):=\left\{u: J \rightarrow X ; u \in C\left(J_{k}, X\right), k=\right.$ $0,1, \ldots, m$, and the right limit $u\left(t_{k}^{+}\right)$exists for $\left.k=1,2, \ldots, m\right\}$ with the norm $\|u\|_{P C}:=$ $\sup _{t \in J}\|u(t)\|$ and $P C^{1}(J, X):=\left\{u: J \rightarrow X ; u \in C^{1}\left(J_{k}, X\right), k=0,1, \ldots, m\right.$, and the right limits $u\left(t_{k}^{+}\right), u^{\prime}\left(t_{k}^{+}\right)$exist for $\left.k=1,2, \ldots, m\right\}$ with the norm $\|u\|_{1}:=\|u\|_{P C^{1}}=\max \left\{\sup _{t \in J}\|u(t)\|\right.$, $\left.\sup _{t \in J}\left\|u^{\prime}(t)\right\|\right\}$. Obviously, $P C(J, X)$ and $P C^{1}(J, X)$ are Banach spaces.

Let us recall the following known definitions. For more details see [4].

Let $g \in L^{1}([a, \infty), \mathbf{R})$, the set of all integrable functions from $[a, \infty)$ to $\mathbf{R}$.

Definition 2.1 ([4]) The fractional integral of order $q$ with the lower limit $a$ for a function $g(t)$ is defined as

$$
{ }_{a} I_{t}^{q} g(t)=\frac{1}{\Gamma(q)} \int_{a}^{t}(t-s)^{q-1} g(s) d s, \quad t>a, q>0
$$

provided the right side is point-wise defined on $[a, \infty)$, where $\Gamma(\cdot)$ is the gamma function. 
Definition 2.2 ([4]) Riemann-Liouville derivative of order $q$ with the lower limit $a$ for a function $g(t)$ can be written as

$$
{ }_{a}^{L} D_{t}^{q} g(t)=\frac{1}{\Gamma(n-q)} \frac{d^{n}}{d t^{n}} \int_{a}^{t}(t-s)^{n-q-1} g(s) d s, \quad t>a, n-1<q<n .
$$

Definition 2.3 ([4]) The Caputo derivative of order $q$ for the function $g(t)$ can be written as

$$
{ }_{a}^{c} D_{t}^{q} g(t)={ }_{a}^{L} D_{t}^{q}\left(g(t)-\sum_{k=0}^{n-1} \frac{(t-a)^{k}}{k !} g^{(k)}(a)\right), \quad t>a, n-1<q<n .
$$

\section{Remark 2.4}

(1) If $g(t) \in C^{n}[a, \infty)$, then

$$
\begin{aligned}
{ }_{a}^{c} D_{t}^{q} g(t) & =\frac{1}{\Gamma(n-q)} \int_{a}^{t}(t-s)^{n-q-1} g^{(n)}(s) d s \\
& ={ }_{a} I_{t}^{n-q} g^{(n)}(t), \quad t>a, n-1<q<n .
\end{aligned}
$$

(2) The Caputo derivative of a constant is equal to zero.

Moreover, from the definition of Caputo derivative, we can obtain the following auxiliary results.

Lemma 2.5 For $q>0$, the general solution of fractional differential equation ${ }_{a}^{c} D_{t}^{q} u(t)=0$ is given by

$$
u(t)=c_{0}+c_{1}(t-a)+c_{2}(t-a)^{2}+\cdots+c_{n-1}(t-a)^{n-1},
$$

where $c_{i} \in \mathbf{R}, i=0,1,2, \ldots, n-1(n=[q]+1)$ and $[q]$ denotes the integer part of the real number $q$.

In view of Lemma 2.5 , it follows that the result holds.

Lemma 2.6 Let $q>0$, then

$$
{ }_{a} I_{t}^{q}\left({ }_{a}^{c} D_{t}^{q} u\right)(t)=u(t)+c_{0}+c_{1}(t-a)+c_{2}(t-a)^{2}+\cdots+c_{n-1}(t-a)^{n-1},
$$

where $c_{i} \in \mathbf{R}, i=0,1,2, \ldots, n-1, n=[q]+1$.

Definition 2.7 A function $u \in P C^{1}(J, X)$ is said to be a solution of (1.1)-(1.4) if $u$ satisfies the equation ${ }^{c} D_{t}^{q} u(t)=f(t, u(t))$ on $J^{\prime}$, and conditions $\Delta u\left(t_{k}\right)=I_{k}\left(u\left(t_{k}\right)\right), \Delta u^{\prime}\left(t_{k}\right)=\widetilde{I}_{k}\left(u\left(t_{k}\right)\right)$, $k=1,2, \ldots, m$ and $u(0)+u(1)=\alpha \int_{0}^{1} q_{1}(u(s)) d s, u^{\prime}(0)+u^{\prime}(1)=\beta \int_{0}^{1} q_{2}(u(s)) d s$. 
Lemma 2.8 Let $h: J \rightarrow X$ be continuous. A function $u: J \rightarrow X$ is a solution of the following fractional integral equation:

$$
u(t)=\left\{\begin{array}{c}
\frac{1}{\Gamma(q)} \int_{0}^{t}(t-s)^{q-1} h(s) d s-\frac{1}{2} \sum_{i=1}^{m}\left(\frac{1}{\Gamma(q)} \int_{t_{i-1}}^{t_{i}}\left(t_{i}-s\right)^{q-1} h(s) d s+I_{i}\left(u\left(t_{i}\right)\right)\right) \\
\quad-\frac{1}{4} \sum_{i=1}^{m}\left(1+2\left(t-t_{i}\right)\right)\left(\frac{1}{\Gamma(q-1)} \int_{t_{i-1}}^{t_{i}}\left(t_{i}-s\right)^{q-2} h(s) d s+\widetilde{I}_{i}\left(u\left(t_{i}\right)\right)\right) \\
\quad-\frac{1}{2 \Gamma(q)} \int_{t_{m}}^{1}(1-s)^{q-1} h(s) d s+\frac{1-2 t}{4 \Gamma(q-1)} \int_{t_{m}}^{1}(1-s)^{q-2} h(s) d s \\
\quad+\frac{\alpha}{2} \int_{0}^{1} q_{1}(u(s)) d s-\frac{\beta(1-2 t)}{4} \int_{0}^{1} q_{2}(u(s)) d s, \quad t \in J_{0}, \\
\frac{1}{\Gamma(q)} \int_{t_{k}}^{t}(t-s)^{q-1} h(s) d s+\frac{1}{2} \sum_{i=1}^{k}\left(\frac{1}{\Gamma(q)} \int_{t_{i-1}}^{t_{i}}\left(t_{i}-s\right)^{q-1} h(s) d s+I_{i}\left(u\left(t_{i}\right)\right)\right) \\
\quad-\frac{1}{2} \sum_{i=k+1}^{m}\left(\frac{1}{\Gamma(q)} \int_{t_{i-1}}^{t_{i}}\left(t_{i}-s\right)^{q-1} h(s) d s+I_{i}\left(u\left(t_{i}\right)\right)\right) \\
\quad+\frac{1}{2} \sum_{i=1}^{k}\left(\frac{1}{\Gamma(q-1)} \int_{t_{i-1}}^{t_{i}}\left(t_{i}-s\right)^{q-2} h(s) d s+\widetilde{I}_{i}\left(u\left(t_{i}\right)\right)\right) \cdot\left(t-t_{i}\right) \\
\quad-\frac{1}{2} \sum_{i=k+1}^{m}\left(\frac{1}{\Gamma(q-1)} \int_{t_{i-1}}^{t_{i}}\left(t_{i}-s\right)^{q-2} h(s) d s+\widetilde{I}_{i}\left(u\left(t_{i}\right)\right)\right) \cdot\left(t-t_{i}\right) \\
\quad-\frac{1}{4} \sum_{i=1}^{m}\left(\frac{1}{\Gamma(q-1)} \int_{t_{i-1}}^{t_{i}}\left(t_{i}-s\right)^{q-2} h(s) d s+\widetilde{I}_{i}\left(u\left(t_{i}\right)\right)\right) \\
\quad-\frac{1}{2 \Gamma(q)} \int_{t_{m}}^{1}(1-s)^{q-1} h(s) d s+\frac{1-2 t}{4 \Gamma(q-1)} \int_{t_{m}}^{1}(1-s)^{q-2} h(s) d s \\
\quad+\frac{\alpha}{2} \int_{0}^{1} q_{1}(u(s)) d s-\frac{\beta(1-2 t)}{4} \int_{0}^{1} q_{2}(u(s)) d s, \quad t \in J_{k}, k=1,2, \ldots, m,
\end{array}\right.
$$

if and only if $u(t)$ is a solution of the problem

$$
\begin{aligned}
& { }^{c} D_{t}^{q} u(t)=h(t), \quad t \in J^{\prime}:=J \backslash\left\{t_{1}, t_{2}, \ldots, t_{m}\right\}, J=[0,1], \\
& \Delta u\left(t_{k}\right)=I_{k}\left(u\left(t_{k}\right)\right), \quad \Delta u^{\prime}\left(t_{k}\right)=\widetilde{I}_{k}\left(u\left(t_{k}\right)\right), \quad k=1,2, \ldots, m, \\
& u(0)+u(1)=\alpha \int_{0}^{1} q_{1}(u(s)) d s, \\
& u^{\prime}(0)+u^{\prime}(1)=\beta \int_{0}^{1} q_{2}(u(s)) d s .
\end{aligned}
$$

Proof Let $u$ be the solution of (2.2)-(2.5). If $t \in J_{0}$, then Lemma 2.6 implies that

$$
\begin{aligned}
& u(t)=\frac{1}{\Gamma(q)} \int_{0}^{t}(t-s)^{q-1} h(s) d s+a_{0}+b_{0} t \\
& u^{\prime}(t)=\frac{1}{\Gamma(q-1)} \int_{0}^{t}(t-s)^{q-2} h(s) d s+b_{0},
\end{aligned}
$$

for some $a_{0}, b_{0} \in \mathbf{R}$. Obviously, $u(0)=a_{0}, u^{\prime}(0)=b_{0}$.

If $t \in J_{1}$, then Lemma 2.6 implies that

$$
\begin{aligned}
& u(t)=\frac{1}{\Gamma(q)} \int_{t_{1}}^{t}(t-s)^{q-1} h(s) d s+c_{0}+c_{1}\left(t-t_{1}\right), \\
& u^{\prime}(t)=\frac{1}{\Gamma(q-1)} \int_{t_{1}}^{t}(t-s)^{q-2} h(s) d s+c_{1}
\end{aligned}
$$

for some $c_{0}, c_{1} \in \mathbf{R}$. Thus, we have

$$
\begin{aligned}
& u\left(t_{1}^{-}\right)=\frac{1}{\Gamma(q)} \int_{0}^{t_{1}}\left(t_{1}-s\right)^{q-1} h(s) d s+a_{0}+b_{0} t_{1}, \\
& u\left(t_{1}^{+}\right)=c_{0},
\end{aligned}
$$




$$
\begin{aligned}
& u^{\prime}\left(t_{1}^{-}\right)=\frac{1}{\Gamma(q-1)} \int_{0}^{t_{1}}\left(t_{1}-s\right)^{q-2} h(s) d s+b_{0}, \\
& u^{\prime}\left(t_{1}^{+}\right)=c_{1} .
\end{aligned}
$$

Applying the impulsive condition (2.3), we derive

$$
\begin{aligned}
& c_{0}=\frac{1}{\Gamma(q)} \int_{0}^{t_{1}}\left(t_{1}-s\right)^{q-1} h(s) d s+a_{0}+b_{0} t_{1}+I_{1}\left(u\left(t_{1}\right)\right), \\
& c_{1}=\frac{1}{\Gamma(q-1)} \int_{0}^{t_{1}}\left(t_{1}-s\right)^{q-2} h(s) d s+b_{0}+\widetilde{I}_{1}\left(u\left(t_{1}\right)\right) .
\end{aligned}
$$

Hence, for $t \in J_{1}$,

$$
\begin{aligned}
u(t)= & \frac{1}{\Gamma(q)} \int_{t_{1}}^{t}(t-s)^{q-1} h(s) d s+\frac{1}{\Gamma(q)} \int_{0}^{t_{1}}\left(t_{1}-s\right)^{q-1} h(s) d s \\
& +\frac{1}{\Gamma(q-1)} \int_{0}^{t_{1}}\left(t_{1}-s\right)^{q-2} h(s) d s \cdot\left(t-t_{1}\right)+I_{1}\left(u\left(t_{1}\right)\right) \\
& +\widetilde{I}_{1}\left(u\left(t_{1}\right)\right)\left(t-t_{1}\right)+a_{0}+b_{0} t .
\end{aligned}
$$

If $t \in J_{2}$, then Lemma 2.6 implies that

$$
\begin{aligned}
& u(t)=\frac{1}{\Gamma(q)} \int_{t_{2}}^{t}(t-s)^{q-1} h(s) d s+d_{0}+d_{1}\left(t-t_{2}\right), \\
& u^{\prime}(t)=\frac{1}{\Gamma(q-1)} \int_{t_{2}}^{t}(t-s)^{q-2} h(s) d s+d_{1},
\end{aligned}
$$

for some $d_{0}, d_{1} \in \mathbf{R}$. Thus, we have

$$
\begin{aligned}
u\left(t_{2}^{-}\right)= & \frac{1}{\Gamma(q)} \int_{t_{1}}^{t_{2}}\left(t_{2}-s\right)^{q-1} h(s) d s+\frac{1}{\Gamma(q)} \int_{0}^{t_{1}}\left(t_{1}-s\right)^{q-1} h(s) d s \\
& +\frac{1}{\Gamma(q-1)} \int_{0}^{t_{1}}\left(t_{1}-s\right)^{q-2} h(s) d s \cdot\left(t_{2}-t_{1}\right)+I_{1}\left(u\left(t_{1}\right)\right) \\
& +\widetilde{I}_{1}\left(u\left(t_{1}\right)\right)\left(t_{2}-t_{1}\right)+a_{0}+b_{0} t_{2}, \\
u\left(t_{2}^{+}\right)= & d_{0}, \\
u^{\prime}\left(t_{2}^{-}\right)= & \frac{1}{\Gamma(q-1)} \int_{t_{1}}^{t_{2}}\left(t_{2}-s\right)^{q-2} h(s) d s+\frac{1}{\Gamma(q-1)} \int_{0}^{t_{1}}\left(t_{1}-s\right)^{q-2} h(s) d s \\
& +b_{0}+\widetilde{I}_{1}\left(u\left(t_{1}\right)\right), \\
u^{\prime}\left(t_{2}^{+}\right)= & d_{1} .
\end{aligned}
$$

Applying the impulsive condition (2.3), we obtain

$$
\begin{aligned}
d_{0}= & \frac{1}{\Gamma(q)} \int_{t_{1}}^{t_{2}}\left(t_{2}-s\right)^{q-1} h(s) d s+\frac{1}{\Gamma(q)} \int_{0}^{t_{1}}\left(t_{1}-s\right)^{q-1} h(s) d s \\
& +\frac{1}{\Gamma(q-1)} \int_{0}^{t_{1}}\left(t_{1}-s\right)^{q-2} h(s) d s \cdot\left(t_{2}-t_{1}\right)+I_{1}\left(u\left(t_{1}\right)\right)
\end{aligned}
$$




$$
\begin{aligned}
& +\widetilde{I}_{1}\left(u\left(t_{1}\right)\right)\left(t_{2}-t_{1}\right)+I_{2}\left(u\left(t_{2}\right)\right)+a_{0}+b_{0} t_{2}, \\
d_{1}= & \frac{1}{\Gamma(q-1)} \int_{t_{1}}^{t_{2}}\left(t_{2}-s\right)^{q-2} h(s) d s+\frac{1}{\Gamma(q-1)} \int_{0}^{t_{1}}\left(t_{1}-s\right)^{q-2} h(s) d s \\
& +b_{0}+\widetilde{I}_{1}\left(u\left(t_{1}\right)\right)+\widetilde{I}_{2}\left(u\left(t_{2}\right)\right) .
\end{aligned}
$$

Hence, for $t \in J_{2}$,

$$
\begin{aligned}
u(t)= & \frac{1}{\Gamma(q)} \int_{t_{2}}^{t}(t-s)^{q-1} h(s) d s+\frac{1}{\Gamma(q)} \int_{t_{1}}^{t_{2}}\left(t_{2}-s\right)^{q-1} h(s) d s \\
& +\frac{1}{\Gamma(q)} \int_{0}^{t_{1}}\left(t_{1}-s\right)^{q-1} h(s) d s+\frac{1}{\Gamma(q-1)} \int_{0}^{t_{1}}\left(t_{1}-s\right)^{q-2} h(s) d s \cdot\left(t-t_{1}\right) \\
& +\frac{1}{\Gamma(q-1)} \int_{t_{1}}^{t_{2}}\left(t_{2}-s\right)^{q-2} h(s) d s \cdot\left(t-t_{2}\right)+I_{1}\left(u\left(t_{1}\right)\right)+I_{2}\left(u\left(t_{2}\right)\right) \\
& +\widetilde{I}_{1}\left(u\left(t_{1}\right)\right)\left(t-t_{1}\right)+\widetilde{I}_{2}\left(u\left(t_{2}\right)\right)\left(t-t_{2}\right)+a_{0}+b_{0} t .
\end{aligned}
$$

By repeating the process, for $t \in J_{k}$, we have

$$
\begin{aligned}
u(t)= & \frac{1}{\Gamma(q)} \int_{t_{k}}^{t}(t-s)^{q-1} h(s) d s \\
& +\sum_{i=1}^{k}\left(\frac{1}{\Gamma(q)} \int_{t_{i-1}}^{t_{i}}\left(t_{i}-s\right)^{q-1} h(s) d s+I_{i}\left(u\left(t_{i}\right)\right)\right) \\
& +\sum_{i=1}^{k}\left(\frac{1}{\Gamma(q-1)} \int_{t_{i-1}}^{t_{i}}\left(t_{i}-s\right)^{q-2} h(s) d s+\widetilde{I}_{i}\left(u\left(t_{i}\right)\right)\right) \cdot\left(t-t_{i}\right) \\
& +a_{0}+b_{0} t, \quad t \in J_{k} .
\end{aligned}
$$

Now, applying the boundary conditions (2.4), (2.5) to (2.2), we get

$$
\begin{aligned}
b_{0}= & -\frac{1}{2} \sum_{i=1}^{m}\left(\frac{1}{\Gamma(q-1)} \int_{t_{i-1}}^{t_{i}}\left(t_{i}-s\right)^{q-2} h(s) d s+\widetilde{I}_{i}\left(u\left(t_{i}\right)\right)\right) \\
& +\frac{\beta}{2} \int_{0}^{1} q_{2}(u(s)) d s-\frac{1}{2 \Gamma(q-1)} \int_{t_{m}}^{1}(1-s)^{q-2} h(s) d s, \\
a_{0}= & -\frac{1}{2 \Gamma(q)} \int_{t_{m}}^{1}(1-s)^{q-1} h(s) d s+\frac{1}{4 \Gamma(q-1)} \int_{t_{m}}^{1}(1-s)^{q-2} h(s) d s \\
& -\frac{1}{2} \sum_{i=1}^{m}\left(\frac{1}{\Gamma(q)} \int_{t_{i-1}}^{t_{i}}\left(t_{i}-s\right)^{q-1} h(s) d s+I_{i}\left(u\left(t_{i}\right)\right)\right) \\
& -\frac{1}{2} \sum_{i=1}^{m}\left(\frac{1}{2}-t_{i}\right)\left(\frac{1}{\Gamma(q-1)} \int_{t_{i-1}}^{t_{i}}\left(t_{i}-s\right)^{q-2} h(s) d s+\widetilde{I}_{i}\left(u\left(t_{i}\right)\right)\right) \\
& +\frac{\alpha}{2} \int_{0}^{1} q_{1}(u(s)) d s-\frac{\beta}{4} \int_{0}^{1} q_{2}(u(s)) d s .
\end{aligned}
$$

Now, it is clear that (2.6)-(2.9) imply (2.1). 
Conversely, if we assume that $u$ satisfies (2.1), by a direct computation, it follows that the solution given by (2.1) satisfies (2.2)-(2.5). This completes the proof.

Next, we recall that the Hausdorff measure of noncompactness $\chi(\cdot)$ on each bounded subset $\Omega$ of Banach space $Y$ is defined by

$$
\chi(\Omega)=\inf \{\varepsilon>0 ; \Omega \text { has a finite } \varepsilon \text {-net in } Y\} .
$$

This measure of noncompactness satisfies some basic properties as follows.

Lemma 2.9 ([22]) Let $Y$ be a Banach space and let $U, V \subseteq Y$ be bounded. Then

(1) $\chi(U)=0$ if and only if $U$ is precompact;

(2) $\chi(U)=\chi(\bar{U})=\chi(\operatorname{conv} U)$, where $\bar{U}$ and conv $U$ mean the closure and convex hull of $U$, respectively;

(3) $\chi(U) \leq \chi(V)$ if $U \subseteq V$;

(4) $\chi(U \cup V) \leq \max \{\chi(U), \chi(V)\}$;

(5) $\chi(U+V) \leq \chi(U)+\chi(V)$, where $U+V=\{x+y ; x \in U, y \in V\}$;

(6) $\chi(\lambda U)=|\lambda| \chi(U)$, for any $\lambda \in \mathbf{R}$;

(7) if the map $Q: D(Q) \subseteq Y \rightarrow Z$ is Lipschitz continuous with constant $k$, then $\chi(Q \Omega) \leq k \chi(\Omega)$ for any bounded subset $\Omega \subseteq D(Q)$, where $Z$ is a Banach space.

Definition 2.10 A continuous map $Q: W \subseteq Y \rightarrow Y$ is said to be a $\chi$-contraction if there exists a positive constant $v<1$ such that $\chi(Q U) \leq v \cdot \chi(U)$ for any bounded closed subset $U \subseteq W$.

Theorem 2.11 (Darbo-Sadovskii [22]) If $U \subseteq Y$ is bounded closed and convex, the continuous map $\mathcal{F}: U \rightarrow U$ is a $\chi$-contraction, then the map $\mathcal{F}$ has at least one fixed point in $U$.

In Section 3, we will use the above fixed point theorem to obtain main result. To this end, we present the following assertion about $\chi$-estimates for a multivalued integral (Theorem 4.2 .3 of [23]).

Let $2^{Y}$ be the family of all nonempty subset of $Y, G:[0, d] \rightarrow 2^{Y}$ be a multifunction. It is called:

(i) integrable, if it admits a Bochner integrable selection $\varrho:[0, d] \rightarrow Y, \varrho(t) \in G(t)$ for a.e. $t \in[0, d]$

(ii) integrably bounded, if there exists a function $\vartheta \in L^{1}([0, d], Y)$ such that

$$
\|G(t)\|:=\sup \{\|\varrho\| ; \varrho \in G(t)\} \leq \vartheta(t) \quad \text { a.e. } t \in[0, d] .
$$

Proposition 2.12 For an integrable, integrably bounded multifunction $G:[0, d] \rightarrow 2^{X}$ where $X$ is a separable Banach space, let

$$
\chi(G(t)) \leq m(t), \quad \text { for a.e. } t \in[0, d]
$$

where $m \in L_{+}^{1}([0, d])$. Then $\chi\left(\int_{0}^{t} G(s) d s\right) \leq \int_{0}^{t} m(s) d s$ for all $t \in[0, d]$. 
To end this section, we introduce the following $P C^{1}$-type Arzela-Ascoli theorem ([24], Theorem 2.1) which will be used in Section 3.

Theorem 2.13 Let $Y$ be a Banach space and $\mathcal{W} \subset P C^{1}(J, Y)$. If the following conditions are satisfied:

(i) $\mathcal{W}$ is a uniformly bounded subset of $P C^{1}(J, Y)$;

(ii) $\mathcal{W}$ is equicontinuous in $\left(t_{k}, t_{k+1}\right), k=0,1,2, \ldots, m$, where $t_{0}=0, t_{m+1}=1$;

(iii) $\mathcal{W}(t)=\left\{u(t) ; u \in \mathcal{W}, t \in J \backslash\left\{t_{1}, \ldots, t_{m}\right\}\right\}, \mathcal{W}\left(t_{k}^{+}\right)=\left\{u\left(t_{k}^{+}\right) ; u \in \mathcal{W}\right\}$ and $\mathcal{W}\left(t_{k}^{-}\right)=\left\{u\left(t_{k}^{-}\right) ; u \in \mathcal{W}\right\}$ are relatively compact subsets of $Y$,

then $\mathcal{W}$ is a relatively compact subset of $P C^{1}(J, Y)$.

\section{Main results}

In this section, we will discuss the existence of solutions to (1.1)-(1.4). For this end, we consider the following assumptions.

(H1) (i) $f: J \times X \rightarrow X$ satisfies $f(\cdot, v): J \rightarrow X$ is measurable for all $v \in X$ and $f(t, \cdot): X \rightarrow X$ is continuous for a.e. $t \in J$, and there exists a function $\mu \in L^{\frac{1}{\sigma_{1}}}\left(J, \mathbf{R}^{+}\right)\left(\sigma_{1} \in\left(0, \frac{q-1}{2}\right)\right)$ such that

$$
\|f(t, v)\| \leq \mu(t) .
$$

(ii) For any bounded set $D \subset X$, there exists a function $\eta \in L^{\frac{1}{\sigma_{2}}}\left(J, \mathbf{R}^{+}\right)$ $\left(\sigma_{2} \in(0, q-1)\right)$ such that

$$
\chi(f(t, D)) \leq \eta(t) \chi(D) .
$$

(H2) The functions $I_{k}, \widetilde{I}_{k}: X \rightarrow X(k=1,2, \ldots, m)$ are continuous and there exist positive constants $l_{k}, \widetilde{l}_{k}$ such that

$$
\begin{aligned}
& \left\|I_{k}(u)-I_{k}(v)\right\| \leq l_{k}\|u-v\|, \quad u, v \in X, \\
& \left\|\widetilde{I}_{k}(u)-\widetilde{I}_{k}(v)\right\| \leq \widetilde{l}_{k}\|u-v\|, \quad u, v \in X .
\end{aligned}
$$

(H3) There exist constants $M_{q_{1}}, M_{q_{2}} \in \mathbf{R}^{+}$such that

$$
\left\|q_{i}\left(u_{1}\right)-q_{i}\left(u_{2}\right)\right\| \leq M_{q_{i}}\left\|u_{1}-u_{2}\right\|, \quad u_{1}, u_{2} \in X, i=1,2 .
$$

Theorem 3.1 Assume that (H1)-(H3) are satisfied, then there exists a solution of (1.1)-(1.4) on J provided that

$$
\begin{aligned}
\kappa:= & \frac{(3 m+1) q-m+5}{2 \Gamma(q)}\left(\frac{1-\sigma_{2}}{q-\sigma_{2}-1}\right)^{1-\sigma_{2}}\|\eta\|_{L^{\frac{1}{\sigma_{2}}}} \\
& +\sum_{i=1}^{m} \frac{2 l_{i}+3 \widetilde{l}_{i}}{4}+\frac{\alpha M_{q_{1}}+\beta M_{q_{2}}}{2}<1 .
\end{aligned}
$$


Proof Consider the operator $\mathcal{F}: P C^{1}(J, X) \rightarrow P C^{1}(J, X)$ defined by

$$
(\mathcal{F} u)(t)=\left\{\begin{array}{c}
\frac{1}{\Gamma(q)} \int_{0}^{t}(t-s)^{q-1} f(s, u(s)) d s \\
\quad-\frac{1}{2} \sum_{i=1}^{m}\left(\frac{1}{\Gamma(q)} \int_{t_{i-1}}^{t_{i}}\left(t_{i}-s\right)^{q-1} f(s, u(s)) d s+I_{i}\left(u\left(t_{i}\right)\right)\right) \\
\quad-\frac{1}{4} \sum_{i=1}^{m}\left(1+2\left(t-t_{i}\right)\right)\left(\frac{1}{\Gamma(q-1)} \int_{t_{i-1}}^{t_{i}}\left(t_{i}-s\right)^{q-2} f(s, u(s)) d s+\widetilde{I}_{i}\left(u\left(t_{i}\right)\right)\right) \\
\quad-\frac{1}{2 \Gamma(q)} \int_{t_{m}}^{1}(1-s)^{q-1} f(s, u(s)) d s+\frac{1-2 t}{4 \Gamma(q-1)} \int_{t_{m}}^{1}(1-s)^{q-2} f(s, u(s)) d s \\
\quad+\frac{\alpha}{2} \int_{0}^{1} q_{1}(u(s)) d s-\frac{\beta(1-2 t)}{4} \int_{0}^{1} q_{2}(u(s)) d s, \quad t \in J_{0}, \\
\frac{1}{\Gamma(q)} \int_{t_{k}}^{t}(t-s)^{q-1} f(s, u(s)) d s \\
\quad+\frac{1}{2} \sum_{i=1}^{k}\left(\frac{1}{\Gamma(q)} \int_{t_{i-1}}^{t_{i}}\left(t_{i}-s\right)^{q-1} f(s, u(s)) d s+I_{i}\left(u\left(t_{i}\right)\right)\right) \\
\quad-\frac{1}{2} \sum_{i=k+1}^{m}\left(\frac{1}{\Gamma(q)} \int_{t_{i-1}}^{t_{i}}\left(t_{i}-s\right)^{q-1} f(s, u(s)) d s+I_{i}\left(u\left(t_{i}\right)\right)\right) \\
\quad+\frac{1}{2} \sum_{i=1}^{k}\left(\frac{1}{\Gamma(q-1)} \int_{t_{i-1}}^{t_{i}}\left(t_{i}-s\right)^{q-2} f(s, u(s)) d s+\widetilde{I}_{i}\left(u\left(t_{i}\right)\right)\right) \cdot\left(t-t_{i}\right) \\
\left.\quad-\frac{1}{2} \sum_{i=k+1}^{m} \frac{1}{\Gamma(q-1)} \int_{t_{i-1}}^{t_{i}}\left(t_{i}-s\right)^{q-2} f(s, u(s)) d s+\widetilde{I}_{i}\left(u\left(t_{i}\right)\right)\right) \cdot\left(t-t_{i}\right) \\
\quad-\frac{1}{4} \sum_{i=1}^{m}\left(\frac{1}{\Gamma(q-1)} \int_{t_{i-1}}^{t_{i}}\left(t_{i}-s\right)^{q-2} f(s, u(s)) d s+\widetilde{I}_{i}\left(u\left(t_{i}\right)\right)\right) \\
\quad-\frac{1}{2 \Gamma(q)} \int_{t_{m}}^{1}(1-s)^{q-1} f(s, u(s)) d s+\frac{1-2 t}{4 \Gamma(q-1)} \int_{t_{m}}^{1}(1-s)^{q-2} f(s, u(s)) d s \\
\quad+\frac{\alpha}{2} \int_{0}^{1} q_{1}(u(s)) d s-\frac{\beta(1-2 t)}{4} \int_{0}^{1} q_{2}(u(s)) d s, \quad t \in J_{k}, k=1,2, \ldots, m .
\end{array}\right.
$$

Clearly, $\mathcal{F}$ is well defined and the fixed point of $\mathcal{F}$ is the solution of problem (1.1)-(1.4) by Lemma 2.8 .

The operator $\mathcal{F}$ can be rewritten in the form $\mathcal{F}=\mathcal{F}_{1}+\mathcal{F}_{2}$, for $t \in J_{k}(k=0,1,2, \ldots, m)$, where

$$
\begin{aligned}
\left(\mathcal{F}_{1} u\right)(t)= & \frac{1}{\Gamma(q)} \int_{t_{k}}^{t}(t-s)^{q-1} f(s, u(s)) d s \\
& +\frac{1}{2 \Gamma(q)} \sum_{i=1}^{k} \int_{t_{i-1}}^{t_{i}}\left(t_{i}-s\right)^{q-1} f(s, u(s)) d s \\
& -\frac{1}{2 \Gamma(q)} \sum_{i=k+1}^{m} \int_{t_{i-1}}^{t_{i}}\left(t_{i}-s\right)^{q-1} f(s, u(s)) d s \\
& +\frac{1}{2 \Gamma(q-1)} \sum_{i=1}^{k}\left(\int_{t_{i-1}}^{t_{i}}\left(t_{i}-s\right)^{q-2} f(s, u(s)) d s\right) \cdot\left(t-t_{i}\right) \\
& -\frac{1}{2 \Gamma(q-1)} \sum_{i=k+1}^{m}\left(\int_{t_{i-1}}^{t_{i}}\left(t_{i}-s\right)^{q-2} f(s, u(s)) d s\right) \cdot\left(t-t_{i}\right) \\
& -\frac{1}{4 \Gamma(q-1)} \sum_{i=1}^{m} \int_{t_{i-1}}^{t_{i}}\left(t_{i}-s\right)^{q-2} f(s, u(s)) d s \\
& -\frac{1}{2 \Gamma(q)} \int_{t_{m}}^{1}(1-s)^{q-1} f(s, u(s)) d s+\frac{1-2 t}{4 \Gamma(q-1)} \int_{t_{m}}^{1}(1-s)^{q-2} f(s, u(s)) d s, \\
\left(\mathcal{F}_{2} u\right)(t)= & \frac{1}{2} \sum_{i=1}^{k} I_{i}\left(u\left(t_{i}\right)\right)-\frac{1}{2} \sum_{i=k+1}^{m} I_{i}\left(u\left(t_{i}\right)\right)-\frac{1}{4} \sum_{i=1}^{m} \widetilde{I}_{i}\left(u\left(t_{i}\right)\right) \\
& +\frac{1}{2} \sum_{i=1}^{k} \widetilde{I}_{i}\left(u\left(t_{i}\right)\right) \cdot\left(t-t_{i}\right)-\frac{1}{2} \sum_{i=k+1}^{m} \widetilde{I}_{i}\left(u\left(t_{i}\right)\right) \cdot\left(t-t_{i}\right) \\
& +\frac{\alpha}{2} \int_{0}^{1} q_{1}(u(s)) d s-\frac{\beta(1-2 t)}{4} \int_{0}^{1} q 2(u(s)) d s . \\
&
\end{aligned}
$$


Then

$$
\begin{aligned}
\left(\mathcal{F}_{1} u\right)^{\prime}(t)= & \frac{1}{\Gamma(q-1)} \int_{t_{k}}^{t}(t-s)^{q-2} f(s, u(s)) d s \\
& +\frac{1}{2 \Gamma(q-1)} \sum_{i=1}^{k}\left(\int_{t_{i-1}}^{t_{i}}\left(t_{i}-s\right)^{q-2} f(s, u(s)) d s\right) \\
& -\frac{1}{2 \Gamma(q-1)} \sum_{i=k+1}^{m+1}\left(\int_{t_{i-1}}^{t_{i}}\left(t_{i}-s\right)^{q-2} f(s, u(s)) d s\right) \\
\left(\mathcal{F}_{2} u\right)^{\prime}(t)= & \frac{1}{2} \sum_{i=1}^{k} \widetilde{I}_{i}\left(u\left(t_{i}\right)\right)-\frac{1}{2} \sum_{i=k+1}^{m} \widetilde{I}_{i}\left(u\left(t_{i}\right)\right) \\
& +\frac{\beta}{2} \int_{0}^{1} q_{2}(u(s)) d s .
\end{aligned}
$$

Let $\left\{u_{n}\right\}$ be a sequence such that $u_{n} \rightarrow u$ in $P C^{1}(J, X)$ as $n \rightarrow \infty$. Since $f$ satisfies (H1)(i), for almost every $t \in J$, we get $f\left(t, u_{n}(t)\right) \rightarrow f(t, u(t))$ as $n \rightarrow \infty$. Moreover, $\| f\left(t, u_{n}(t)\right)-$ $f(t, u(t)) \| \leq 2 \mu(t)$.

It follows from Lebesgue's dominated convergence theorem that

$$
\left\|\left(\mathcal{F}_{1} u_{n}\right)(t)-\left(\mathcal{F}_{1} u\right)(t)\right\|_{1} \rightarrow 0, \quad \text { as } n \rightarrow \infty .
$$

Moreover, noting that $(\mathrm{H} 2)$ and $(\mathrm{H} 3)$, we have $\left\|\left(\mathcal{F}_{2} u_{n}\right)(t)-\left(\mathcal{F}_{2} u\right)(t)\right\|_{1} \rightarrow 0$ as $n \rightarrow \infty$. Now we can see that $\mathcal{F}$ is continuous.

Let $B_{r}=\left\{u \in P C^{1}(J, X) ;\|u\|_{1} \leq r\right\}$, where $r \geq \frac{C_{0}}{1-C_{1}}$ and

$$
\begin{aligned}
C_{0}= & \frac{\left(1-\sigma_{1}\right)^{1-\sigma_{1}} \cdot\|\mu\|_{L^{\frac{1}{\sigma_{1}}}}}{\Gamma(q)\left(q-\sigma_{1}-1\right)^{1-\sigma_{1}}} \cdot \frac{(3 m+1) q-m+5}{4} \\
& +\sum_{i=1}^{m} \frac{2\left\|I_{i}(0)\right\|+3\left\|\widetilde{I}_{i}(0)\right\|}{4}+\frac{\alpha\left\|q_{1}(0)\right\|+\beta\left\|q_{2}(0)\right\|}{2}, \\
C_{1}= & \sum_{i=1}^{m} \frac{2 l_{i}+3 \widetilde{l}_{i}}{4}+\frac{\alpha M_{q_{1}}+\beta M_{q_{2}}}{2} .
\end{aligned}
$$

For $u \in B_{r}, i=1,2, \ldots, m+1$, using (H1)(i) and the Hölder inequality, we have

$$
\begin{aligned}
\left\|\int_{t_{i-1}}^{t_{i}}\left(t_{i}-s\right)^{q-1} f(s, u(s)) d s\right\| & \leq\left(\int_{t_{i-1}}^{t_{i}}\left(t_{i}-s\right)^{\frac{q-1}{1-\sigma_{1}}} d s\right)^{1-\sigma_{1}}\left(\int_{t_{i-1}}^{t_{i}}(\mu(s))^{\frac{1}{\sigma_{1}}} d s\right)^{\sigma_{1}} \\
& <\left(\frac{1-\sigma_{1}}{q-\sigma_{1}}\right)^{1-\sigma_{1}}\|\mu\|_{L^{\frac{1}{\sigma_{1}}}}, \\
\left\|\int_{t_{i-1}}^{t_{i}}\left(t_{i}-s\right)^{q-2} f(s, u(s)) d s\right\| & \leq\left(\int_{t_{i-1}}^{t_{i}}\left(t_{i}-s\right)^{\frac{q-2}{1-\sigma_{1}}} d s\right)^{1-\sigma_{1}}\left(\int_{t_{i-1}}^{t_{i}}(\mu(s))^{\frac{1}{\sigma_{1}}} d s\right)^{\sigma_{1}} \\
& <\left(\frac{1-\sigma_{1}}{q-\sigma_{1}-1}\right)^{1-\sigma_{1}}\|\mu\|_{L^{\frac{1}{\sigma_{1}}}} .
\end{aligned}
$$


Therefore

$$
\begin{aligned}
& \left\|\left(\mathcal{F}_{1} u\right)(t)\right\|<\frac{\left(1-\sigma_{1}\right)^{1-\sigma_{1}} \cdot\|\mu\|_{L^{\frac{1}{\sigma_{1}}}}}{\Gamma(q)\left(q-\sigma_{1}-1\right)^{1-\sigma_{1}}} \cdot \frac{(3 m+1) q-m+5}{4}, \\
& \left\|\left(\mathcal{F}_{1} u\right)^{\prime}(t)\right\|<\frac{\left(1-\sigma_{1}\right)^{1-\sigma_{1}} \cdot\|\mu\|_{L^{\frac{1}{\sigma_{1}}}}}{\Gamma(q-1)\left(q-\sigma_{1}-1\right)^{1-\sigma_{1}}} \cdot \frac{m+3}{2},
\end{aligned}
$$

then

$$
\left\|\left(\mathcal{F}_{1} u\right)(t)\right\|_{1} \leq \frac{\left(1-\sigma_{1}\right)^{1-\sigma_{1}} \cdot\|\mu\|_{L^{\frac{1}{\sigma_{1}}}}}{\Gamma(q)\left(q-\sigma_{1}-1\right)^{1-\sigma_{1}}} \cdot \frac{(3 m+1) q-m+5}{4} .
$$

Moreover, by (H2) and (H3), we obtain

$$
\begin{aligned}
\left\|\left(\mathcal{F}_{2} u\right)(t)\right\|< & \sum_{i=1}^{m} \frac{\left(2 l_{i}+3 \widetilde{l}_{i}\right)}{4}\left\|u\left(t_{i}\right)\right\|+\frac{\alpha M_{q_{1}}+\beta M_{q_{2}}}{2} \int_{0}^{1}\|u(s)\| d s \\
& +\sum_{i=1}^{m} \frac{2\left\|I_{i}(0)\right\|+3\left\|\widetilde{I}_{i}(0)\right\|}{4}+\frac{\alpha\left\|q_{1}(0)\right\|+\beta\left\|q_{2}(0)\right\|}{2}
\end{aligned}
$$

and

$$
\left\|\left(\mathcal{F}_{2} u\right)^{\prime}(t)\right\| \leq \frac{1}{2} \sum_{i=1}^{m} \widetilde{l}_{i}\left\|u\left(t_{i}\right)\right\|+\frac{\beta M_{q_{2}}}{2} \int_{0}^{1}\|u(s)\| d s+\frac{1}{2} \sum_{i=1}^{m}\left\|\widetilde{I}_{i}(0)\right\|+\frac{\beta\left\|q_{2}(0)\right\|}{2},
$$

therefore

$$
\begin{aligned}
\left\|\left(\mathcal{F}_{2} u\right)(t)\right\|_{1} \leq & \left(\sum_{i=1}^{m} \frac{2 l_{i}+3 \widetilde{l}_{i}}{4}+\frac{\alpha M_{q_{1}}+\beta M_{q_{2}}}{2}\right) r+\sum_{i=1}^{m} \frac{2\left\|I_{i}(0)\right\|+3\left\|\widetilde{I}_{i}(0)\right\|}{4} \\
& +\frac{\alpha\left\|q_{1}(0)\right\|+\beta\left\|q_{2}(0)\right\|}{2} .
\end{aligned}
$$

From (3.2) and (3.3), we have $\|(\mathcal{F} u)(t)\|_{1} \leq C_{0}+C_{1} r \leq r$. This shows that $\mathcal{F} B_{r} \subset B_{r}$.

Let $u \in B_{r}$, for any $t_{k}<\tau_{2}<\tau_{1} \leq t_{k+1}$, we have

$$
\begin{aligned}
& \left\|\frac{1}{\Gamma(q)} \int_{t_{k}}^{\tau_{1}}\left(\tau_{1}-s\right)^{q-1} f(s, u(s)) d s-\frac{1}{\Gamma(q)} \int_{t_{k}}^{\tau_{2}}\left(\tau_{2}-s\right)^{q-1} f(s, u(s)) d s\right\| \\
& \quad \leq \frac{1}{\Gamma(q)} \int_{t_{k}}^{\tau_{2}}\left[\left(\tau_{1}-s\right)^{q-1}-\left(\tau_{2}-s\right)^{q-1}\right] \mu(s) d s+\frac{1}{\Gamma(q)} \int_{\tau_{2}}^{\tau_{1}}\left(\tau_{1}-s\right)^{q-1} \mu(s) d s \\
& \quad \rightarrow 0, \quad \text { as } \tau_{2} \rightarrow \tau_{1} .
\end{aligned}
$$

Therefore,

$$
\begin{aligned}
& \left\|(\mathcal{F} u)\left(\tau_{1}\right)-(\mathcal{F} u)\left(\tau_{2}\right)\right\| \\
& \quad \leq\left\|\frac{1}{\Gamma(q)} \int_{t_{k}}^{\tau_{1}}\left(\tau_{1}-s\right)^{q-1} f(s, u(s)) d s-\frac{1}{\Gamma(q)} \int_{t_{k}}^{\tau_{2}}\left(\tau_{2}-s\right)^{q-1} f(s, u(s)) d s\right\|
\end{aligned}
$$




$$
\begin{aligned}
& +\frac{\left(\tau_{1}-\tau_{2}\right)}{2}\left[\sum_{i=1}^{m}\left\|\widetilde{I}_{i}\left(u\left(t_{i}\right)\right)\right\|+\beta \int_{0}^{1}\left\|q_{2}(u(s))\right\| d s\right] \\
& +\frac{\left(\tau_{1}-\tau_{2}\right)}{2 \Gamma(q-1)} \sum_{i=1}^{m+1} \int_{t_{i-1}}^{t_{i}}\left(t_{i}-s\right)^{q-2} \mu(s) d s \\
& \rightarrow 0, \quad \text { as } \tau_{2} \rightarrow \tau_{1} .
\end{aligned}
$$

Moreover, from the Hölder inequality, we have

$$
\begin{aligned}
& \frac{1}{\Gamma(q-1)}\left\|\int_{t_{k}}^{\tau_{2}}\left[\left(\tau_{1}-s\right)^{q-2}-\left(\tau_{2}-s\right)^{q-2}\right] \mu(s) d s\right\| \\
& \quad \leq \frac{1}{\Gamma(q-1)}\left(\int_{t_{k}}^{\tau_{2}}\left|\left(\tau_{1}-s\right)^{q-2}-\left(\tau_{2}-s\right)^{q-2}\right|^{\frac{1}{1-\sigma_{1}}} d s\right)^{1-\sigma_{1}} \cdot\|\mu\|_{L^{\frac{1}{\sigma_{1}}}} \\
& \quad=\frac{2-q}{\Gamma(q-1)} \cdot\|\mu\|_{L^{\frac{1}{\sigma_{1}}}}\left(\int_{t_{k}}^{\tau_{2}}\left[\int_{\tau_{2}}^{\tau_{1}}(\zeta-s)^{q-3} d \zeta\right]^{\frac{1}{1-\sigma_{1}}} d s\right)^{1-\sigma_{1}} \\
& \quad \leq M\left(\int_{t_{k}}^{\tau_{2}}\left(\left(\tau_{2}-s\right)^{\theta}-\left(\tau_{1}-s\right)^{\theta}\right) d s\right)^{1-\sigma_{1}} \\
& \quad \leq \frac{M}{(1+\theta)^{1-\sigma_{1}}}\left[\left(\tau_{1}-\tau_{2}\right)^{1+\theta}-\left(\tau_{1}-t_{k}\right)^{1+\theta}+\left(\tau_{2}-t_{k}\right)^{1+\theta}\right]^{1-\sigma_{1}} \\
& \quad \rightarrow 0, \quad \text { as } \tau_{2} \rightarrow \tau_{1},
\end{aligned}
$$

where $M>0$ is a constant and $\theta=\frac{q-2-\sigma_{1}}{1-\sigma_{1}}$. Similarly,

$$
\begin{aligned}
\int_{\tau_{2}}^{\tau_{1}}\left(\tau_{1}-s\right)^{q-2} \mu(s) d s & \leq\left(\int_{\tau_{2}}^{\tau_{1}}\left(\tau_{1}-s\right)^{\frac{q-2}{1-\sigma_{1}}} d s\right)^{1-\sigma_{1}} \cdot\|\mu\|_{L^{\frac{1}{\sigma_{1}}}} \\
& =\left(\frac{1-\sigma_{1}}{q-1-\sigma_{1}}\right)^{1-\sigma_{1}}\left(\tau_{1}-\tau_{2}\right)^{q-1-\sigma_{1}} \cdot\|\mu\|_{L^{\frac{1}{\sigma_{1}}}} .
\end{aligned}
$$

Thus,

$$
\begin{aligned}
& \left\|(\mathcal{F} u)^{\prime}\left(\tau_{1}\right)-(\mathcal{F} u)^{\prime}\left(\tau_{2}\right)\right\| \\
& \quad \leq \frac{1}{\Gamma(q-1)}\left\|\int_{t_{k}}^{\tau_{1}}\left(\tau_{1}-s\right)^{q-2} f(s, u(s)) d s-\int_{t_{k}}^{\tau_{2}}\left(\tau_{2}-s\right)^{q-2} f(s, u(s)) d s\right\| \\
& \leq \frac{1}{\Gamma(q-1)}\left\|\int_{t_{k}}^{\tau_{2}}\left[\left(\tau_{1}-s\right)^{q-2}-\left(\tau_{2}-s\right)^{q-2}\right] \mu(s) d s\right\|+\frac{1}{\Gamma(q-1)} \int_{\tau_{2}}^{\tau_{1}}\left(\tau_{1}-s\right)^{q-2} \mu(s) d s \\
& \quad \rightarrow 0, \quad \text { as } \tau_{2} \rightarrow \tau_{1} .
\end{aligned}
$$

This shows that the set $\left\{(\mathcal{F} u)(\cdot) ; u \in B_{r}\right\}$ is equicontinuous.

If $W \subset P C^{1}(J, X)$ is bounded and the elements of $W^{\prime}$ are equicontinuous on each $J_{k}=$ $\left(t_{k}, t_{k+1}\right](k=0,1, \ldots, m)$, we can consider the measure of noncompactness $\chi_{p c^{1}}$ on the space $P C^{1}(J, X)$ in the following way:

$$
\chi_{p c^{1}}(W)=\max \left\{\max _{0 \leq k \leq m} \chi\left(W\left(J_{k}\right)\right), \max _{0 \leq k \leq m} \chi\left(W^{\prime}\left(J_{k}\right)\right)\right\} .
$$


Let $\Omega \subset B_{r}$ be a nonempty, bounded set. Clearly, we can see

$$
\begin{aligned}
& \chi\left(\int_{t_{k}}^{t}(t-s)^{q-1} f(s, \Omega(s)) d s\right) \\
& \quad \leq \int_{t_{k}}^{t}(t-s)^{q-1} \eta(s) \chi(\Omega(s)) d s, \quad t \in J_{k}, k=0,1,2, \ldots, m, \\
& \chi\left(\int_{t_{i-1}}^{t_{i}}\left(t_{i}-s\right)^{q-1} f(s, \Omega(s)) d s\right) \\
& \quad \leq \int_{t_{i-1}}^{t_{i}}\left(t_{i}-s\right)^{q-1} \eta(s) \chi(\Omega(s)) d s, \quad i=1,2, \ldots, m+1 .
\end{aligned}
$$

Moreover, for $i=1,2, \ldots, m+1$, we set

$$
\widetilde{\mathcal{F}}_{i}(\Omega)=\left\{\int_{t_{i-1}}^{t_{i}}\left(t_{i}-s\right)^{q-2} f(s, u(s)) d s ; u \in \Omega\right\} .
$$

Consider the multifunction $s \in\left[t_{i-1}, t_{i}\right] \multimap H_{i}(s)$

$$
H_{i}(s)=\left\{\left(t_{i}-s\right)^{q-2} f(s, u(s)) ; u \in \Omega\right\}, \quad i=1,2, \ldots, m+1 .
$$

Obviously, $H_{i}$ is integrable and from (H1)(i) it follows that $H_{i}$ is integrably bounded. Moreover, noting that (H1)(ii), we have the following estimate for a.e. $s \in\left[t_{i-1}, t_{i}\right], i=$ $1,2, \ldots, m+1$

$$
\chi\left(H_{i}(s)\right)=\chi\left(\left\{\left(t_{i}-s\right)^{q-2} f(s, u(s)) ; u \in \Omega\right\}\right) \leq\left(t_{i}-s\right)^{q-2} \eta(s) \chi(\Omega(s)) .
$$

Applying Proposition 2.12, we obtain

$$
\chi\left(\widetilde{\mathcal{F}}_{i}(\Omega)\right)=\chi\left(\int_{t_{i-1}}^{t_{i}} H_{i}(s) d s\right) \leq \int_{t_{i-1}}^{t_{i}}\left(t_{i}-s\right)^{q-2} \eta(s) \chi(\Omega(s)) d s .
$$

Therefore, combining with (3.4), (3.5) and the Hölder inequality, we have

$$
\begin{aligned}
\chi\left(\left(\mathcal{F}_{1} \Omega\right)(t)\right) \leq & \frac{1}{\Gamma(q)} \int_{t_{k}}^{t}(t-s)^{q-1} \eta(s) \chi(\Omega(s)) d s \\
& +\frac{1}{2 \Gamma(q)} \sum_{i=1}^{m} \int_{t_{i-1}}^{t_{i}}\left(t_{i}-s\right)^{q-1} \eta(s) \chi(\Omega(s)) d s \\
& +\frac{3}{4 \Gamma(q-1)} \sum_{i=1}^{m} \int_{t_{i-1}}^{t_{i}}\left(t_{i}-s\right)^{q-2} \eta(s) \chi(\Omega(s)) d s \\
& +\frac{1}{2 \Gamma(q)} \int_{t_{m}}^{1}(1-s)^{q-1} \eta(s) \chi(\Omega(s)) d s \\
& +\frac{1}{4 \Gamma(q-1)} \int_{t_{m}}^{1}(1-s)^{q-2} \eta(s) \chi(\Omega(s)) d s \\
< & \frac{(3 m+1) q-m+5}{2 \Gamma(q)}\left(\frac{1-\sigma_{2}}{q-\sigma_{2}-1}\right)^{1-\sigma_{2}}\|\eta\|_{L^{\frac{1}{\sigma_{2}}}} \cdot \chi_{p c^{1}}(\Omega) .
\end{aligned}
$$


Similarly,

$$
\begin{aligned}
\chi\left(\left(\mathcal{F}_{1} \Omega\right)^{\prime}(t)\right) \leq & \frac{1}{\Gamma(q-1)} \int_{t_{k}}^{t}(t-s)^{q-2} \eta(s) \chi(\Omega(s)) d s \\
& +\frac{1}{2 \Gamma(q-1)} \sum_{i=1}^{m+1} \int_{t_{i-1}}^{t_{i}}\left(t_{i}-s\right)^{q-2} \eta(s) \chi(\Omega(s)) d s \\
\leq & \frac{(m+3)}{2 \Gamma(q-1)}\left(\frac{1-\sigma_{2}}{q-\sigma_{2}-1}\right)^{1-\sigma_{2}}\|\eta\|_{L^{\frac{1}{\sigma_{2}}}} \cdot \chi_{p c^{1}}(\Omega) .
\end{aligned}
$$

Since $\mathcal{F}_{1} \Omega$ and $\left(\mathcal{F}_{1} \Omega\right)^{\prime}$ are equicontinuous on every $J_{k}$, by Proposition 7.3 of [25], we find that

$$
\begin{aligned}
\chi_{p c^{1}}\left(\mathcal{F}_{1} \Omega\right) & =\max \left\{\max _{0 \leq k \leq m} \chi\left(\left(\mathcal{F}_{1} \Omega\right)\left(J_{k}\right)\right), \max _{0 \leq k \leq m} \chi\left(\left(\mathcal{F}_{1} \Omega\right)^{\prime}\left(J_{k}\right)\right)\right\} \\
& =\max \left\{\max _{0 \leq k \leq m} \max _{t \in J_{k}} \chi\left(\left(\mathcal{F}_{1} \Omega\right)(t)\right), \max _{0 \leq k \leq m} \max _{t \in J_{k}} \chi\left(\left(\mathcal{F}_{1} \Omega\right)^{\prime}(t)\right)\right\} .
\end{aligned}
$$

Then, according to inequalities (3.6) and (3.7), we have

$$
\chi_{p c^{1}}\left(\mathcal{F}_{1} \Omega\right)<\frac{(3 m+1) q-m+5}{2 \Gamma(q)}\left(\frac{1-\sigma_{2}}{q-\sigma_{2}-1}\right)^{1-\sigma_{2}}\|\eta\|_{L^{\frac{1}{\sigma_{2}}}} \cdot \chi_{p c^{1}}(\Omega) .
$$

Moreover, by (H2) and (H3), for any $u, v \in B_{r}$,

$$
\left\|\left(\mathcal{F}_{2} u\right)(t)-\left(\mathcal{F}_{2} v\right)(t)\right\|_{1} \leq C_{1}\|u-v\|_{1}, \quad t \in J
$$

This means that $\mathcal{F}_{2}$ is Lipschitz continuous with Lipschitz constant $C_{1}$. It follows from (7) in Lemma 2.9 that

$$
\chi_{p c^{1}}\left(\mathcal{F}_{2} \Omega\right) \leq C_{1} \chi_{p c^{1}}(\Omega)
$$

Therefore, from (3.8) and (3.9), we get

$$
\chi_{p c^{1}}(\mathcal{F} \Omega) \leq \chi_{p c^{1}}\left(\mathcal{F}_{1} \Omega\right)+\chi_{p c^{1}}\left(\mathcal{F}_{2} \Omega\right) \leq \kappa \chi_{p c^{1}}(\Omega)
$$

Hence, $\mathcal{F}$ is a $\chi$-contraction on $B_{r}$ by Definition 2.10. According to Theorem 2.11, the operator $\mathcal{F}$ has at least one fixed point $u$ in $B_{r}$, which is a solution of problem (1.1)-(1.4). This ends the proof.

When $X=\mathbf{R}$, we rely on Schauder's fixed point theorem, which gives an existence result for solutions of problem (1.1)-(1.4) under the following assumptions.

$\left(\mathrm{H}^{\prime}\right) f: J \times \mathbf{R} \rightarrow \mathbf{R}$ is jointly continuous, and there exists a function $\mu \in L^{\frac{1}{\sigma_{1}}}\left(J, \mathbf{R}^{+}\right)\left(\sigma_{1} \in\right.$ $\left.\left(0, \frac{q-1}{2}\right)\right)$ such that $|f(t, v)| \leq \mu(t)$.

(H2') The functions $I_{k}, \widetilde{I}_{k}: \mathbf{R} \rightarrow \mathbf{R}$ are continuous and there exist positive constants $l_{k}, \widetilde{l}_{k}$ such that $\left|I_{k}(u)-I_{k}(v)\right| \leq l_{k}|u-v|$ and $\left|\widetilde{I}_{k}(u)-\widetilde{I}_{k}(v)\right| \leq \widetilde{l}_{k}|u-v|, u, v \in \mathbf{R}, k=1,2, \ldots, m$.

$\left(\mathrm{H}^{\prime}\right)$ There exist constants $M_{q_{1}}, M_{q_{2}} \in \mathbf{R}^{+}$such that $\left|q_{i}\left(u_{1}\right)-q_{i}\left(u_{2}\right)\right| \leq M_{q_{i}}\left|u_{1}-u_{2}\right|, u_{1}, u_{2} \in$ $\mathbf{R}, i=1,2$. 
Theorem 3.2 Assume that the assumptions (H1')-(H3') hold. Then problem (1.1)-(1.4) has at least one solution on J provided that

$$
\widetilde{\kappa}:=\sum_{i=1}^{m} \frac{2 l_{i}+3 \widetilde{l}_{i}}{4}+\frac{\alpha M_{q_{1}}+\beta M_{q_{2}}}{2}<1
$$

Proof Let $\mathcal{F}: P C^{1}(J, \mathbf{R}) \rightarrow P C^{1}(J, \mathbf{R})$ be defined as in the proof of Theorem 3.1. In the proof of Theorem 3.1, we can see that $\mathcal{F}$ is continuous, $\mathcal{F}$ maps bounded sets $B_{r}$ into bounded sets and equicontinuous sets. Then we can deduce that $\mathcal{F}$ is compact on $B_{r}$ as a result of the $P C^{1}$-type Arzela-Ascoli theorem (see Theorem 2.13 in the case of $X=\mathbf{R}$ ).

As a consequence of Schauder's fixed point theorem, we conclude that $\mathcal{F}$ has a fixed point, that is, problem (1.1)-(1.4) has at least one solution on $\mathbf{R}$. The proof is complete.

\section{Applications}

In this section, we give two examples to illustrate the usefulness of our main results.

Example 4.1 Let $X=L^{2}([0, \pi])$. Consider the following impulsive integral boundary problem:

$$
\left\{\begin{array}{l}
\partial_{t}^{q} v(t, x)=a(t) \cos (|v(t, x)|), \quad t \in J^{\prime}=[0,1] \backslash\left\{t_{1}, t_{2}, \ldots, t_{m}\right\}, \\
\triangle v\left(t_{k}, x\right)=\int_{0}^{\pi} \rho_{k}(x, y) d y \cdot \sin ^{2}\left(v\left(t_{k}, x\right)\right), \quad k=1,2, \ldots, m, \\
\triangle v^{\prime}\left(t_{k}, x\right)=\frac{1}{2^{k}} \cdot \frac{\left|v\left(t_{k}, x\right)\right|}{1+\left|v\left(t_{k}, x\right)\right|}, \quad k=1,2, \ldots, m, \\
v(0, x)+v(1, x)=\int_{0}^{1} b(s) e^{-|v(s, x)|} d s, \\
v^{\prime}(0, x)+v^{\prime}(1, x)=\int_{0}^{1} c(s) \cdot \frac{|v(s, x)|}{1+|v(s, x)|} d s,
\end{array}\right.
$$

where $q \in(1,2), x \in[0, \pi], a(t) \in L^{\frac{1}{\sigma}}\left(J, \mathbf{R}^{+}\right), \sigma \in\left(0, \frac{q-1}{2}\right), b(t), c(t)$ are bounded functions on $\mathbf{R}$ such that $|b(t)| \leq b^{*},|c(t)| \leq c^{*}$, the functions $\rho_{k}(x, y), y \in[0, \pi](k=1,2, \ldots, m)$ are measurable and there exists a constant $\bar{N}$ such that $\int_{0}^{\pi}\left\|\rho_{k}(x, y)\right\| d y \leq \bar{N}$.

For $t \in[0,1], x \in[0, \pi], k=1,2, \ldots, m$, we set

$$
\begin{aligned}
& u(t)(x)=v(t, x), \\
& f(t, u(t))(x)=a(t) \cos (|u(t)(x)|), \\
& I_{k}\left(u\left(t_{k}\right)\right)(x)=\int_{0}^{\pi} \rho_{k}(x, y) d y \cdot \sin ^{2}\left(u\left(t_{k}\right)(x)\right), \quad k=1,2, \ldots, m, \\
& \widetilde{I}_{k}\left(u\left(t_{k}\right)\right)(x)=\frac{1}{2^{k}} \cdot \frac{\left|u\left(t_{k}\right)(x)\right|}{1+\left|u\left(t_{k}\right)(x)\right|}, \quad k=1,2, \ldots, m, \\
& q_{1}(u(t)(x))=b(t) e^{-|u(t)(x)|}, \\
& q_{2}(u(t)(x))=c(t) \cdot \frac{|u(t)(x)|}{1+|u(t)(x)|} .
\end{aligned}
$$

Then we can rewrite (4.1) in the abstract form (1.1)-(1.4).

Obviously, we have $\|f(t, u(t))(x)\| \leq a(t)$ for $t \in[0,1]$. For any $u_{1}, u_{2} \in X$,

$$
\left\|f\left(t, u_{1}(t)\right)-f\left(t, u_{2}(t)\right)\right\| \leq a(t)\left\|u_{1}(t)-u_{2}(t)\right\|,
$$

which implies that for any bounded set $D \subset X, \chi(f(t, D)) \leq a(t) \chi(D), t \in[0,1]$. 
Moreover, for any $u, \tilde{u} \in X$,

$$
\begin{aligned}
& \left\|I_{k}\left(u\left(t_{k}\right)\right)-I_{k}\left(\widetilde{u}\left(t_{k}\right)\right)\right\| \leq 2 \bar{N}\left\|u\left(t_{k}\right)-\widetilde{u}\left(t_{k}\right)\right\|, \\
& \left\|\widetilde{I}_{k}\left(u\left(t_{k}\right)\right)-\widetilde{I}_{k}\left(\widetilde{u}\left(t_{k}\right)\right)\right\| \leq \frac{1}{2^{k}}\left\|u\left(t_{k}\right)-\widetilde{u}\left(t_{k}\right)\right\|, \\
& \left\|q_{1}(u(t))-q_{1}(\widetilde{u}(t))\right\| \leq b^{*}\|u(t)-\widetilde{u}(t)\|, \\
& \left\|q_{2}(u(t))-q_{2}(\widetilde{u}(t))\right\| \leq c^{*}\|u(t)-\widetilde{u}(t)\| .
\end{aligned}
$$

Suppose further that

$$
\frac{(3 m+1) q-m+5}{2 \Gamma(q)}\left(\frac{1-\sigma}{q-\sigma-1}\right)^{1-\sigma}\|a\|_{L^{\frac{1}{\sigma}}}+m \bar{N}+\frac{3}{4}\left(1-\frac{1}{2^{m}}\right)+\frac{b^{*}+c^{*}}{2}<1,
$$

then problem (4.1) has at least a solution by Theorem 3.1.

Example 4.2 Let $X=\mathbf{R}$. Consider the following impulsive anti-periodic boundary problem of fractional order:

$$
\left\{\begin{array}{l}
{ }^{c} D_{t}^{\frac{3}{2}} u(t)=e^{t} \arctan (u(t))+t \int_{0}^{t} e^{-3|u(s)|} d s, \quad t \in[0,1] \backslash\left\{\frac{1}{3}\right\}, \\
\triangle u\left(\frac{1}{3}\right)=\frac{e^{-\left|u\left(\frac{1}{3}\right)\right|}}{10+e^{t}}, \\
\triangle u^{\prime}\left(\frac{1}{3}\right)=\frac{2+\left|u\left(\frac{1}{3}\right)\right|}{7+\left|u\left(\frac{1}{3}\right)\right|} \\
u(0, x)+u(1, x)=0, \\
u^{\prime}(0, x)+u^{\prime}(1, x)=0 .
\end{array}\right.
$$

Set $q=\frac{3}{2}, \sigma=\frac{1}{6}$ and $f(t, u(t))=e^{t} \arctan (u(t))+t \int_{0}^{t} e^{-3|u(s)|} d s$. Obviously, $\|f(t, u(t))\|<$ $\frac{\pi}{2}\left(e^{t}+t^{2}\right) \in L^{6}([0,1], \mathbf{R})$. Moreover, $l_{1}=\frac{1}{10}, \widetilde{l}_{1}=\frac{5}{49}$. It can be found that $\widetilde{\kappa}=\frac{1}{20}+\frac{3 \times 5}{4 \times 49}<1$. Therefore, due to the fact that all the assumptions of Theorem 3.2 hold, problem (4.2) has a solution.

\section{Conclusion}

In this paper, a generalized boundary value problem for impulsive fractional differential equations involving Caputo fractional derivative in abstract space has been studied. A reasonable formula and definition of solutions for such problem is introduced. The new existence results are obtained based upon the techniques of measures of noncompactness and the fixed point theory.

\section{Competing interests}

The authors declare that they have no competing interests.

\section{Authors' contributions}

Each of the authors, FL and HW, contributed to each part of this study equally and read and approved the final version of the manuscript.

\section{Acknowledgements}

The authors are grateful to the referee for his/her valuable suggestion. This work was supported partly by the NSF of China (11201413), the NSF of Yunnan Province (2013FB034) and the Educational Commission of Yunnan Province (2012Z010). 


\section{References}

1. Kilbas, AA, Srivastava, HM, Trujillo, JJ: Theory and Applications of Fractional Differential Equations. North-Holland Mathematics Studies, vol. 204. Elsevier, Amsterdam (2006)

2. Lakshmikantham, V, Leela, S, Vasundhara, DJ: Theory of Fractional Dynamic Systems. Cambridge Scientific Publishers, Cambridge (2009)

3. Miller, KS, Ross, B: An Introduction to the Fractional Calculus and Differential Equations. Wiley, New York (1993)

4. Podlubny, I: Fractional Differential Equations. Mathematics in Science and Engineering, vol. 198. Academic Press, San Diego (1999)

5. Diagana, T, Mophou, GM, N'Guérékata, GM: On the existence of mild solutions to some semilinear fractional integro-differential equations. Electron. J. Qual. Theory Differ. Equ. 2010, 58 (2010)

6. Hernandez, E, O'Regan, D, Balachandran, K: On recent developments in the theory of abstract differential equations with fractional derivatives. Nonlinear Anal. 73(10), 3462-3471 (2010)

7. Mophou, GM, N'Guérékata, GM: On some classes of almost automorphic functions and applications to fractional differential equations. Comput. Math. Appl. 59, 1310-1317 (2010)

8. Sakthivel, R, Ren, Y, Mahmudov, NI: On the approximate controllability of semilinear fractional differential systems. Comput. Math. Appl. 62, 1451-1459 (2011)

9. Benchohra, M, Henderson, J, Ntouyas, S: Impulsive Differential Equations and Inclusions. Hindawi Publishing Corporation, New York (2006)

10. Samoilenko, AM, Perestyuk, NA: Impulsive Differential Equations. World Scientific, Singapore (1995)

11. Benchohra, M, Seba, D: Impulsive fractional differential equations in Banach spaces. Electron. J. Qual. Theory Differ. Equ. 2009, 8 (2009) (Special edition I)

12. Balachandran, K, Kiruthika, S: Existence of solutions of abstract fractional impulsive semilinear evolution equations. Electron. J. Qual. Theory Differ. Equ. 2010, 4 (2010)

13. Wang, G, Ahmad, B, Zhang, L, Nieto, JJ: Comments on the concept of existence of solution for impulsive fractional differential equations. Commun. Nonlinear Sci. Numer. Simul. 19, 401-403 (2014)

14. Ahmad, B, Malar, K, Karthikeyan, K: A study of nonlocal problems of impulsive integrodifferential equations with measure of noncompactness. Adv. Differ. Equ. 2013, 205 (2013)

15. Liu, YJ, Ahmad, B: A study of impulsive multiterm fractional differential equations with single and multiple base points and applications. Sci. World J. 2014, Article ID 194346 (2014)

16. Agarwal, RP, Benchohra, M, Hamani, S: A survey on existence results for boundary value problems of nonlinear fractional differential equations and inclusions. Acta Appl. Math. 109, 973-1033 (2010)

17. Ahmad, B, Sivasundaram, S: Existence of solutions for impulsive integral boundary value problems of fractional order. Nonlinear Anal. Hybrid Syst. 4, 134-141 (2010)

18. Ergöen, H, Kiçman, A: Non-local boundary value problems for impulsive fractional integro-differential equations in Banach spaces. Bound. Value Probl. 2012, 145 (2012)

19. Wang, G, Ahmad, B, Zhang, L: Impulsive anti-periodic boundary value problem for nonlinear differential equations of fractional order. Nonlinear Anal. TMA 74, 792-804 (2011)

20. Wang, JR, Zhou, Y, Fečkan, M: On recent developments in the theory of boundary value problems for impulsive fractional differential equations. Comput. Math. Appl. 64, 3008-3020 (2012)

21. Ahmad, B, Nieto, JJ: Existence of solutions for impulsive anti-periodic boundary value problems of fractional order. Taiwan. J. Math. 15(3), 981-993 (2011)

22. Banaś, J, Goebel, K: Measure of Noncompactness in Banach Space. Lecture Notes in Pure and Applied Mathematics, vol. 60. Dekker, New York (1980)

23. Kamenskii, M, Obukhovskii, V, Zecca, P: Condensing Multivalued Maps and Semilinear Differential Inclusions in Banach Spaces. De Gruyter Series in Nonlinear Analysis and Applications, vol. 7. de Gruyter, Berlin (2001)

24. Wei, W, Xiang, X, Peng, Y: Nonlinear impulsive integro-differential equation of mixed type and optimal controls Optimization 55, 141-156 (2006)

25. Deimling, K: Nonlinear Functional Analysis. Springer, Berlin (1985)

10.1186/1687-1847-2014-202

Cite this article as: Li and Wang: Solvability of boundary value problems for impulsive fractional differential equations in Banach spaces. Advances in Difference Equations 2014, 2014:202

\section{Submit your manuscript to a SpringerOpen ${ }^{\ominus}$ journal and benefit from:}

- Convenient online submission

- Rigorous peer review

Immediate publication on acceptance

- Open access: articles freely available online

- High visibility within the field

- Retaining the copyright to your article 